\title{
On Minimizing the Ultimate Ruin Probability of an Insurer by Reinsurance
}

\author{
Christian Kasumo $\mathbb{D}^{1},{ }^{1} \mathrm{Juma} \mathrm{Kasozi}^{2}$, and Dmitry Kuznetsov ${ }^{1}$ \\ ${ }^{1}$ Department of Applied Mathematics and Computational Science, Nelson Mandela African Institution of Science and Technology, \\ P.O. Box 447, Arusha, Tanzania \\ ${ }^{2}$ Department of Mathematics, Makerere University, P.O. Box 7062, Kampala, Uganda
}

Correspondence should be addressed to Christian Kasumo; kasumoc@nm-aist.ac.tz

Received 28 November 2017; Revised 19 January 2018; Accepted 30 January 2018; Published 22 February 2018

Academic Editor: Saeid Abbasbandy

Copyright (C) 2018 Christian Kasumo et al. This is an open access article distributed under the Creative Commons Attribution License, which permits unrestricted use, distribution, and reproduction in any medium, provided the original work is properly cited.

\begin{abstract}
We consider an insurance company whose reserves dynamics follow a diffusion-perturbed risk model. To reduce its risk, the company chooses to reinsure using proportional or excess-of-loss reinsurance. Using the Hamilton-Jacobi-Bellman (HJB) approach, we derive a second-order Volterra integrodifferential equation (VIDE) which we transform into a linear Volterra integral equation (VIE) of the second kind. We then proceed to solve this linear VIE numerically using the block-by-block method for the optimal reinsurance policy that minimizes the ultimate ruin probability for the chosen parameters. Numerical examples with both light- and heavy-tailed distributions are given. The results show that proportional reinsurance increases the survival of the company in both light- and heavy-tailed distributions for the Cramér-Lundberg and diffusion-perturbed models.
\end{abstract}

\section{Introduction}

When the surplus process of an insurance company falls below zero, the company is said to have experienced ruin. Insurance companies customarily take precautions to avoid ruin. These precautions are referred to as control variables and include investments, capital injections or refinancing, portfolio selection, and reinsurance arrangements, to mention but a few. This study focuses on reinsurance as a control measure. Reinsurance, sometimes referred to as "insurance for insurers," is the transfer of risk from a direct insurer (the cedent) to a second insurance carrier (the reinsurer). With reinsurance, the cedent passes on some of its premium income to a reinsurer who, in turn, covers a certain proportion of the claims that occur. It has been argued in the literature that reinsurance plays an important role in risk reduction for cedents in that it offers additional underwriting capacity for them and reduces the probability of a direct insurer's ruin. Apart from helping the cedent to manage financial risk, increase capacity, and achieve marketing goals, reinsurance also benefits policyholders by ensuring availability and affordability of necessary coverage.
Of interest in this paper are those studies which investigate more directly the effect of reinsurance on the ultimate ruin probability. The minimization of the probability of ruin for a company whose claim process evolves according to a Brownian motion with drift and is allowed to invest in a risky asset and to purchase quota-share reinsurance was considered in [1]. In this study, an analytical expression for the minimum ruin probability and the corresponding optimal controls were obtained. Kasozi et al. [2] studied the problem of controlling ultimate ruin probability by quotashare (QS) reinsurance arrangements. Under the assumption that the insurer could invest part of the surplus in a riskfree and risky asset, [2] found that quota-share reinsurance does reduce the probability of ruin and that for chosen parameter values the optimal QS retention $b^{*} \in(0.2,0.4)$. This study also concluded that investment helps insurance companies to reduce their ruin probabilities but that the ruin probabilities increase when stock prices become more volatile. However, while Kasozi et al. [2] considered only quota-share reinsurance, this paper seeks to combine quotashare and excess-of-loss (XL) reinsurance for one and the same insurance portfolio, but in the absence of investment. 
Liu and Yang [3] reconsidered the model in [4] and incorporated a risk-free interest rate. Since closed-form solutions could not be obtained in this case, they provided numerical results for optimal strategies for maximizing the survival probability under different claim-size distribution assumptions. Also using the results in [4], the problem of choosing a combination of investments and optimal dynamic proportional reinsurance to minimize ruin probabilities for an insurance company was investigated in [5] based on a controlled surplus process satisfying the stochastic differential equation $d X_{t}^{A b}=\left(c-c\left(b_{t}\right)+\mu A_{t}\right) d t+\sigma A_{t} d W_{t}-b_{t} d S_{t}$, where $b_{t} \in$ $[0,1]$ is a proportional reinsurance retention at time $t, c\left(b_{t}\right)$ is the dynamic reinsurance premium rate, $\left\{A_{t}\right\}$ is the amount invested in a risky asset at time $t$, and $S_{t}$ is the aggregate claims process. But while [5] uses proportional reinsurance in minimizing ruin probabilities in the Cramér-Lundberg model, this paper considers proportional and excess-of-loss reinsurance in the diffusion-perturbed model.

More recently, taking ruin probability as a risk measure for the insurer, [6] investigated a dynamic optimal reinsurance problem with both fixed and proportional transaction costs for an insurer whose surplus process is modelled by a Brownian motion with positive drift. Under the assumption that the insurer takes noncheap proportional reinsurance, they formulated the problem as a mixed regular control and optimal stopping problem and established that the optimal reinsurance strategy was to never take reinsurance if proportional costs were high and to wait to take the reinsurance when the surplus hits a level. Additionally, they obtained an explicit expression for the survival probability under the optimal reinsurance strategy and found it to be larger than that with the aforementioned strategies. Hu and Zhang [7] introduced a general risk model involving dependence structure with common Poisson shocks. Under a combined quotashare and excess-of-loss reinsurance arrangements, they studied the optimal reinsurance strategy for maximizing the insurer's adjustment coefficient and established that excessof-loss reinsurance was optimal from the insurer's point of view. Zhang and Liang [8] studied the optimal retentions for an insurance company that intends to transfer risk by means of a layer reinsurance treaty. Under the criterion of maximizing the adjustment coefficient, they obtained the closedform expressions of the optimal results for the Brownian motion as well as the compound Poisson risk models and concluded that under the expected value principle excess-ofloss reinsurance is better than any other layer reinsurance strategies while under the variance premium principle pure excess-of-loss reinsurance is no longer the optimal layer reinsurance strategy. Both of these studies, however, used the criterion of maximizing the adjustment coefficient rather than minimizing the insurer's ruin probability.

This paper aims at combining proportional and excessof-loss reinsurance for one and the same insurance portfolio. In proportional or "pro rata" reinsurance, the reinsurer indemnifies the cedent for a predetermined portion of the claims or losses, while in excess-of-loss (XL) reinsurance, which is nonproportional, the reinsurer indemnifies the cedent for all claims or losses or for a specified portion of them, but only if the claim sizes fall within a prespecified band. Excess-of-loss reinsurance has been defined in [9] as "a form of nonproportional reinsurance contract in which an insurer pays insurance claims up to a prefixed retention level and the rest are paid by a reinsurer." Mathematically, given retention level $a$, a claim of size $X$ is divided into the cedent's payment $X \wedge a$ and the reinsurer's payment $X-$ $X \wedge a$. The combination of proportional and excess-of-loss reinsurance has been in fact widely used in the construction of reinsurance models (see, e.g., [10]).

The models in this paper result in Volterra integral equations (VIEs) of the second kind which are solved using the block-by-block method, generally considered as the best of the higher order methods for solving Volterra integral equations of the second kind. The block-by-block methods are essentially extrapolation procedures which produce a block of values at a time. These methods can be of high order and still be self-starting. They do not require special starting procedures, are simple to use, and allow for easy switching of step-size [11].

The rest of the paper is organized as follows. Section 2 presents the formulation of the model and assumptions, followed, in Section 3, by a derivation of the HJB, integrodifferential, and integral equations. In Section 4, we present numerical results for some ruin probability models with reinsurance, using the exponential distribution for small ${ }^{\sim}$ claims and the Pareto distribution for large ones. Some conclusions and possible extensions of this study are given in Section 5.

\section{Model Formulation}

Let $\left(\Omega, \mathscr{F},\left\{\mathscr{F}_{t}\right\}_{t \in \mathbb{R}^{+}}, \mathbb{P}\right)$ be a filtered probability space containing all stochastic objects encountered in this paper and satisfying the usual conditions; that is, $\left\{\mathscr{F}_{t}\right\}_{t \in \mathbb{R}^{+}}$is rightcontinuous and $\mathbb{P}$-complete. In the absence of reinsurance, the surplus of an insurance company is governed by the diffusion-perturbed classical risk process:

$$
U_{t}=u+c t+\sigma W_{t}-\sum_{i=1}^{N_{t}} X_{i}, \quad t \geq 0
$$

where $u=U_{0} \geq 0$ is the initial reserve, $c=(1+\theta) \lambda \mu>$ 0 is the premium rate, $\theta$ is the safety loading, $\left\{N_{t}\right\}$ is a homogeneous Poisson process with intensity $\lambda>0$, and $\left\{X_{i}\right\}$ is an i.i.d. sequence of strictly positive random variables with distribution function $F . S_{t}=\sum_{i=1}^{N_{t}} X_{i}$ is a compound Poisson process representing the cumulative amount of claims paid in the time interval $[0, t]$. The claim arrival process $\left\{N_{t}\right\}$ and claim sizes $\left\{X_{i}\right\}$ are assumed to be independent. Here $\left\{W_{t}\right\}$ is a standard one-dimensional Brownian motion independent of the compound Poisson process $S_{t}$. We assume that $\mathbb{E}\left[X_{i}\right]=$ $\mu<\infty$ and $F(0)=0$. The diffusion term $\sigma W_{t}$ denotes the fluctuations associated with the surplus of the insurance company at time $t$. Without volatility in the surplus and claim amounts, (1) becomes the well-known Cramér-Lundberg model or the classical risk process.

We proceed as in [12] where the insurer took a combination of quota-share and excess-of-loss reinsurance arrangements. Most of the actuarial literature dealing with 
reinsurance as a risk control mechanism only considers pure quota-share or excess-of-loss reinsurance. However, in reality the insurer has the choice of a combination of the two and hence the use of a combination of quota-share and XL reinsurance in this paper. We assume that the reinsurance is cheap, meaning that the reinsurer uses the same safety loading as the insurer. Let the quota-share retention level be $k \in[0,1]$. Then the insurer's aggregate claims, net of quota-share reinsurance, are $k X$. If the company also buys excess-of-loss reinsurance with a retention level $a \in[0, \infty)$, then the insurer's aggregate claims, net of quota-share and excess-of-loss reinsurance, are given by $k X \wedge a$. Given that $\bar{R}$ is a reinsurance strategy combining quota-share and excessof-loss reinsurance, the insurer's controlled surplus process becomes

$$
U_{t}^{\bar{R}}=u+c^{\bar{R}} t+\sigma W_{t}-\sum_{i=1}^{N_{t}} k X_{i} \wedge a,
$$

where the insurance premium $c^{\bar{R}}=c-(1+\theta) \lambda \mathbb{E}\left[\left(k X_{i}-a\right)^{+}\right]$. The controlled surplus process (2) has dynamics

$$
d U_{t}^{\bar{R}}=c^{\bar{R}} d t+\sigma d W_{t}-d\left(\sum_{i=1}^{N_{t}} k X_{i} \wedge a\right) .
$$

The time of ruin is defined as $\tau^{\bar{R}}=\inf \left\{t \geq 0 \mid U_{t}^{\bar{R}}<0\right\}$ and the probability of ultimate ruin is defined as $\psi^{\bar{R}}=\mathbb{P}\left(U_{t}^{\bar{R}}<\right.$ 0 for some $t>0$ ). A reinsurance strategy $\bar{R}$ is said to be admissible if $k \in[0,1]$ and $a \in[0, \infty)$. The objective is to find the quota-share level $k$ and the excess-of-loss retention limit $a$ to minimize the insurer's risk or to maximize the insurer's survival probability. It should be noted that when the retention limit $a$ of the excess-of-loss reinsurance is infinite, then the treaty becomes a pure quota-share reinsurance, while when the quota-share level $k=1$, it becomes a pure excess-ofloss reinsurance treaty. The premium income of the insurance company is nonnegative if $c \geq(1+\theta) \lambda \mathbb{E}\left[(k X-a)^{+}\right]$. Therefore, we will let $\underline{a}$ be the XL retention level at which equality $c=$ $(1+\theta) \lambda \mathbb{E}\left[(\bar{k} X-\underline{a})^{+}\right]$holds.

Define the value function of this problem as

$$
\begin{aligned}
\psi^{\bar{R}}(u) & =\mathbb{P}\left(U_{t} \leq 0 \text { for some } t \geq 0 \mid U_{0}^{\bar{R}}=u\right) \\
& =\mathbb{P}\left(\tau^{\bar{R}}<\infty \mid U_{0}^{\bar{R}}=u\right),
\end{aligned}
$$

where $\psi^{\bar{R}}(u)$ is the probability of ultimate ruin under the policy $\bar{R}$ when the initial surplus is $u$. Then the objective is to find the optimal value function, that is, the minimal ruin probability

$$
\psi(u)=\inf _{(k, a) \in \mathscr{R}} \psi^{\bar{R}}(u)
$$

and optimal policy $(\bar{R})^{*}=\left(k^{*}, a^{*}\right)$ s.t. $\psi^{\bar{R}^{*}}(u)=\psi(u)$. Alternatively, we can find the values of $k^{*}$ and $a^{*}$ which maximize the probability of ultimate survival $\phi(u)=1-\psi(u)$, so that the optimal value function becomes

$$
\phi(u)=\sup _{(k, a) \in \mathscr{R}} \phi^{\bar{R}}(u)
$$

where $\mathscr{R}$ is the set of all reinsurance policies.

\section{HJB, Integrodifferential, and Integral Equations}

Lemma 1. Assume that the survival probability $\phi(u)$ defined by (6) is twice continuously differentiable on $(0, \infty)$. Then $\phi(u)$ satisfies the HJB equation

$$
\begin{aligned}
\sup _{(k, a) \in \mathscr{R}}\left\{\frac{1}{2} \sigma^{2} \phi^{\prime \prime}(u)+c^{\bar{R}} \phi^{\prime}(u)\right. & \\
\left.+\lambda \int_{0}^{u}[\phi(u-k x \wedge a)-\phi(u)] d F(x)\right\}=0, & \\
& u>0,
\end{aligned}
$$

where $\mathscr{R}$ is the set of all reinsurance policies.

Proof. See [13].

We now present the verification theorem which is essential for solving the associated stochastic control problem.

Theorem 2. Suppose $\Phi \in C^{2}$ is an increasing strictly concave function satisfying HJB equation (7) subject to the boundary conditions

$$
\begin{aligned}
\Phi(u)=0 & \text { on } u<0 \\
\Phi(0) & =0 \quad \text { if } \sigma^{2}>0 \\
\lim _{u \rightarrow \infty} \Phi(u) & =1
\end{aligned}
$$

for $0 \leq u<\infty$. Then the maximal survival probability $\phi(u)$ given by (6) coincides with $\Phi$. Furthermore, if $(\bar{R})^{*}=\left(k^{*}, a^{*}\right)$ satisfies

$$
\begin{array}{r}
\frac{1}{2} \sigma^{2} \Phi^{\prime \prime}(u)+c^{\bar{R}^{*}} \Phi^{\prime}(u) \\
+\lambda \int_{0}^{u}\left[\Phi\left(u-k^{*} x \wedge a^{*}\right)-\Phi(u)\right] d F(x)=0 \\
\text { when } 0 \leq u<\infty
\end{array}
$$

then the policy $(\bar{R})^{*}$ is an optimal policy; that is, $\Phi(u)=\phi(u)=$ $\phi^{\bar{R}^{*}}(u)$.

Proof. Let $\bar{R}$ be an arbitrary reinsurance strategy and let $U^{*}$ be the surplus process when $\bar{R}=\bar{R}^{*}$. Choose $n>u$ and define 
$T=\mathscr{T}_{n}=\inf \left\{t \mid U_{t} \notin[0, n]\right\}$. Note that $U_{T \wedge t} \in(-\infty, n]$ because the jumps are downwards. The process

$$
\begin{aligned}
M_{t}^{1} & =\sum_{i=1}^{N_{T \wedge t}}\left[\Phi\left(U_{T_{i}}\right)-\Phi\left(U_{T_{i^{-}}}\right)\right] \\
& -\lambda \int_{0}^{T \wedge t}\left[\int_{0}^{U_{s}} \Phi\left(U_{s}-k x \wedge a\right) d F(x)\right. \\
& \left.-\Phi\left(U_{s}\right)\right] d s
\end{aligned}
$$

is a martingale. We write

$$
\begin{aligned}
& \Phi\left(U_{T \wedge t}\right)=\Phi(u)+\Phi\left(U_{T \wedge t}\right)-\Phi\left(U_{T_{N_{T \wedge t}}}\right) \\
& +\sum_{i=1}^{N_{T \wedge t}}\left[\Phi\left(U_{T_{i^{-}}}\right)-\Phi\left(U_{T_{i-1}}\right)\right]+M_{t}^{1} \\
& +\lambda \int_{0}^{T \wedge t}\left[\int_{0}^{U_{s}} \Phi\left(U_{s}-k x \wedge a\right) d F(x)\right. \\
& \left.\quad-\Phi\left(U_{s}\right)\right] d s .
\end{aligned}
$$

By Itô’s formula,

$$
\begin{aligned}
\Phi\left(U_{T_{i^{-}}}\right)-\Phi\left(U_{T_{i-1}}\right) \\
=\int_{T_{i-1}}^{T_{i}}\left[\frac{1}{2} \sigma^{2} \Phi^{\prime \prime}\left(U_{s}\right)+c^{\bar{R}} \Phi^{\prime}\left(U_{s}\right)\right] d s \\
\quad+\int_{T_{i-1}}^{T_{i}} \sigma \Phi^{\prime}\left(U_{s}\right) d W_{s} .
\end{aligned}
$$

The corresponding result holds for $\Phi\left(U_{T \wedge t}\right)-\Phi\left(U_{T_{N_{T \wedge t}}}\right)$. Thus,

$$
\begin{aligned}
& \Phi\left(U_{T \wedge t}\right)=\Phi(u)+\int_{0}^{T \wedge t}\left[\frac{1}{2} \sigma^{2} \Phi^{\prime \prime}\left(U_{s}\right)+c^{\bar{R}} \Phi^{\prime}\left(U_{s}\right)\right. \\
& \left.+\lambda\left(\int_{0}^{U_{s}} \Phi\left(U_{s}-k x \wedge a\right) d F(x)-\Phi\left(U_{s}\right)\right)\right] d s \\
& \quad+\int_{0}^{T \wedge t} \sigma \Phi^{\prime}\left(U_{s}\right) d W_{s}+M_{t}^{1} .
\end{aligned}
$$

Using HJB equation (7), we find that

$$
\Phi\left(U_{T \wedge t}\right) \leq \Phi(u)+\int_{0}^{T \wedge t} \sigma \Phi^{\prime}\left(U_{s}\right) d W_{s}+M_{t}^{1}
$$

and equality holds for $U^{*}$. Let $\left\{\mathcal{S}_{m}\right\}$ be a localization sequence of the stochastic integral, and set $\mathscr{T}_{n}^{m}=\mathscr{T}_{n} \wedge \mathcal{S}_{m}$. Taking expectations yields

$$
\mathbb{E}\left[\Phi\left(U_{\mathscr{T}_{n}^{m} \wedge t}\right)\right] \leq \Phi(u) .
$$

By bounded convergence, letting $m \rightarrow \infty$ and then $t \rightarrow \infty$, we have $\mathbb{E}\left[\Phi\left(U_{\mathscr{T}_{n}}\right)\right] \leq \Phi(u)$. It turns out that, for $\Phi(0)=0$,

$$
\begin{aligned}
\mathbb{P} & \left(\tau<\mathscr{T}_{n}, U_{\tau}=0\right)+\Phi(n) \mathbb{P}\left(\mathscr{T}_{n}<\tau\right) \\
& =\mathbb{E}\left[\Phi\left(U_{\mathscr{T}_{n}}\right)\right] \leq \Phi(u) .
\end{aligned}
$$

Note that $\mathbb{P}\left(\mathscr{T}_{n}<\tau\right) \geq \phi^{\bar{R}}(u)$. Because there is a strategy with $\phi^{\bar{R}}(u)>0$, it follows that $\Phi(u)$ is bounded. We therefore let $n \rightarrow \infty$, yielding $\mathbb{E}\left[\Phi\left(U_{\tau}\right)\right] \leq \Phi(u)$. In particular, we obtain

$$
\begin{aligned}
\phi^{\bar{R}}(u) \Phi(\infty) & \leq \phi^{\bar{R}}(u) \Phi(\infty)+\mathbb{P}\left(\tau<\infty, U_{\tau}=0\right) \\
& \leq \Phi(u)
\end{aligned}
$$

which simplifies to

$$
\phi^{\bar{R}}(u) \leq \phi^{\bar{R}}(u)+\mathbb{P}\left(\tau<\infty, U_{\tau}=0\right) \leq \Phi(u)
$$

since $\Phi(\infty)=1$. For $U^{*}$ we obtain an equality. In particular, $\left\{\Phi\left(U_{t}^{*}\right)\right\}$ is a martingale. It remains to show that $\mathbb{P}\left(U_{\tau}^{*} \neq\right.$ $0)=1$. Note first from HJB equation (7) that $F(x)$ must be continuous; if not, the integral in (7) is not continuous. Choose $\varepsilon>0$ and consider the strategy $\bar{R}=\bar{R}^{*} \mathbf{1}_{u \geq \varepsilon}$. Let $T_{\varepsilon}=\inf \left\{t \mid U_{t}^{*}<\varepsilon\right\}$. By the martingale property, $\Phi(u)=$ $\Phi(\infty) \mathbb{P}\left(T_{\varepsilon}=\infty\right)+\mathbb{E}\left[\Phi\left(T_{\varepsilon}\right), T_{\varepsilon}<\tau<\infty\right]$ which reduces to

$$
\Phi(u)=\mathbb{P}\left(T_{\varepsilon}=\infty\right)+\mathbb{E}\left[\Phi\left(T_{\varepsilon}\right), T_{\varepsilon}<\tau<\infty\right]
$$

the last term of which is bounded by $\Phi(\varepsilon) \mathbb{P}\left(T_{\varepsilon}<\tau<\right.$ $\infty)$. Since $F(x)$ is continuous, it must converge to zero as $\varepsilon \rightarrow 0$. Because $\mathbb{P}\left(T_{\varepsilon}=\infty\right) \rightarrow \phi^{*}(u)$, it follows that $\Phi(u)=\phi^{*}(u) \Phi(\infty)$ or $\Phi(u)=\phi^{*}(u)=\phi(u)$. That is, $\Phi(u)$ is the optimal value function and $\bar{R}^{*}=(\bar{R})^{*}$ is an optimal policy.

The integrodifferential equation corresponding to optimization problem (6) immediately follows from Theorem 2 as

$$
\begin{aligned}
& \frac{1}{2} \sigma^{2} \phi^{\prime \prime}(u)+c^{\bar{R}} \phi^{\prime}(u) \\
& +\lambda \int_{0}^{u}[\phi(u-k x \wedge a)-\phi(u)] d F(x)=0 \\
& \text { for } 0 \leq u<\infty .
\end{aligned}
$$

This is an integrodifferential equation of Volterra type (VIDE). Solution of this equation will require that it is transformed into a Volterra integral equation (VIE) of the second kind using successive integration by parts. Hence the following theorem is obtained.

Theorem 3. Integrodifferential equation (20) can be represented as a Volterra integral equation of the second kind:

$$
\phi(u)+\int_{0}^{u} K(u, x) \phi(x) d x=h(u),
$$

where

(1) If $u \leq \underline{a}<a$, one has

$$
\begin{aligned}
K(u, x) & =-\frac{\lambda \bar{F}(u-k x)}{c^{\bar{R}}} \\
h(u) & =\phi(0)
\end{aligned}
$$


with $\bar{F}(x)=1-F(x)$, when there is no diffusion (i.e., when $\sigma^{2}=0$ ), and

$$
\begin{gathered}
K(u, x)=\frac{2\left(c^{\bar{R}}+\lambda G(u-k x)-\lambda(u-k x)\right)}{\sigma^{2}} \\
h(u)=u \phi^{\prime}(0) \quad \text { if } \sigma^{2}>0
\end{gathered}
$$

when there is diffusion.

(2) If $\underline{a}<a<u$, one has

$$
\begin{gathered}
K(u, x)=-\frac{\lambda H_{1}(x, u)}{c^{\bar{R}}} \\
h(u)=\phi(0)
\end{gathered}
$$

with

$$
H_{1}(x, u)= \begin{cases}\bar{F}(u-k x) & k x<a \\ 1-(F(k x+a)-F(a)) & k x \geq a\end{cases}
$$

when there is no diffusion, and

$$
\begin{aligned}
K(u, x) & =\frac{2\left(c^{\bar{R}}+\lambda H_{2}(x, u)-\lambda(u-k x)\right)}{\sigma^{2}} \\
h(u) & =u \phi^{\prime}(0) \quad \text { if } \sigma^{2}>0
\end{aligned}
$$

with

$$
\begin{gathered}
H_{2}(x, u)= \begin{cases}G(u-k x) & k x<a \\
(F(k x+a)-F(a))(u-k x) & k x \geq a\end{cases} \\
\text { and } G(x)=\int_{0}^{x} F(v) d v \text { when there is diffusion. }
\end{gathered}
$$

Proof. The proof for the case $u \leq \underline{a}<a$ is similar to the proof of Theorem 2.2 in [14] but with $r=\sigma_{R}^{2}=0, k=1$, and $p=c^{\bar{R}}$. Here, we present the proof for the case $a<a<u$.

Integrating $(20)$ on $[0, z]$ with respect to $u$ gives

$$
\begin{aligned}
0= & \frac{1}{2} \sigma^{2}\left[\phi^{\prime}(z)-\phi^{\prime}(0)\right]+c^{\bar{R}}[\phi(z)-\phi(0)] \\
& -\lambda \int_{0}^{z} \phi(u) d u \\
& +\lambda \int_{0}^{z} \int_{0}^{u} \phi(u-k x \wedge a) f(x) d x d u .
\end{aligned}
$$

To simplify the double integral in (28), we again use integration by parts and Fubini's Theorem (see [13]) to switch the order of integration and change the properties of the convolution integral. Thus,

$$
\begin{aligned}
\int_{0}^{z} & \int_{0}^{u} \phi(u-k x \wedge a) f(x) d x d u \\
= & \int_{0}^{a} F(z-k x) \phi(x) d x \\
& \quad+\int_{a}^{z} \phi(\nu)[F(\nu+a)-F(a)] d \nu,
\end{aligned}
$$

where $v=u-k x$. Substituting into (28) gives

$$
\begin{gathered}
\frac{1}{2} \sigma^{2} \phi^{\prime}(z)-\frac{1}{2} \sigma^{2} \phi^{\prime}(0)+c^{\bar{R}} \phi(z)-c^{\bar{R}} \phi(0) \\
-\lambda \int_{0}^{z} \phi(u) d u+\lambda\left[\int_{0}^{a} F(z-k x) \phi(x) d x\right. \\
\left.+\int_{a}^{z} \phi(\nu)[F(\nu+a)-F(a)] d \nu\right]=0 .
\end{gathered}
$$

Replacing $z$ with $u, v$ and $u$ with $x$, and $F(\nu+a)$ with $F(k x+a)$ gives

$$
\begin{aligned}
& \frac{1}{2} \sigma^{2} \phi^{\prime}(u)-\frac{1}{2} \sigma^{2} \phi^{\prime}(0)+c^{\bar{R}} \phi(u)-c^{\bar{R}} \phi(0) \\
& -\lambda \int_{0}^{u} \phi(x) d x+\lambda \int_{0}^{a} F(u-k x) \phi(x) d x \\
& +\lambda \int_{a}^{u}[F(k x+a)-F(a)] \phi(x) d x=0 .
\end{aligned}
$$

Setting $\sigma^{2}=0$ in (31) yields the case without diffusion

$$
\begin{aligned}
\phi(u) & -\frac{\lambda}{c^{\bar{R}}} \int_{0}^{a} \bar{F}(u-k x) \phi(x) d x \\
& -\frac{\lambda}{c^{\bar{R}}} \int_{a}^{u}[1-(F(k x+a)-F(a))] \phi(x) d x \\
= & \phi(0)
\end{aligned}
$$

from which the kernel is clearly $K(u, x)=-\lambda H_{1}(x, u) / c^{\bar{R}}$ with

$$
H_{1}(x, u)= \begin{cases}\bar{F}(u-k x) & k x<a \\ 1-(F(k x+a)-F(a)) & k x \geq a\end{cases}
$$

and the forcing function is $h(u)=\phi(0)$ as given by (24).

For the case with diffusion, repeated integration by parts of (30) on $[0, u]$ with respect to $z$ yields the desired result.

$$
\begin{aligned}
& \phi(u)+\frac{2}{\sigma^{2}} \int_{0}^{u}\left(c^{\bar{R}}-\lambda(u-k x)\right) \phi(x) d x \\
& +\frac{2 \lambda}{\sigma^{2}}\left[\int_{0}^{a} G(u-k x) \phi(x) d x\right. \\
& \left.+\int_{a}^{u}[F(k x+a)-F(a)](u-k x) \phi(x) d x\right] \\
& =\frac{\sigma^{2}\left(\phi(0)+u \phi^{\prime}(0)\right)+2 c^{\bar{R}} u \phi(0)}{\sigma^{2}}
\end{aligned}
$$




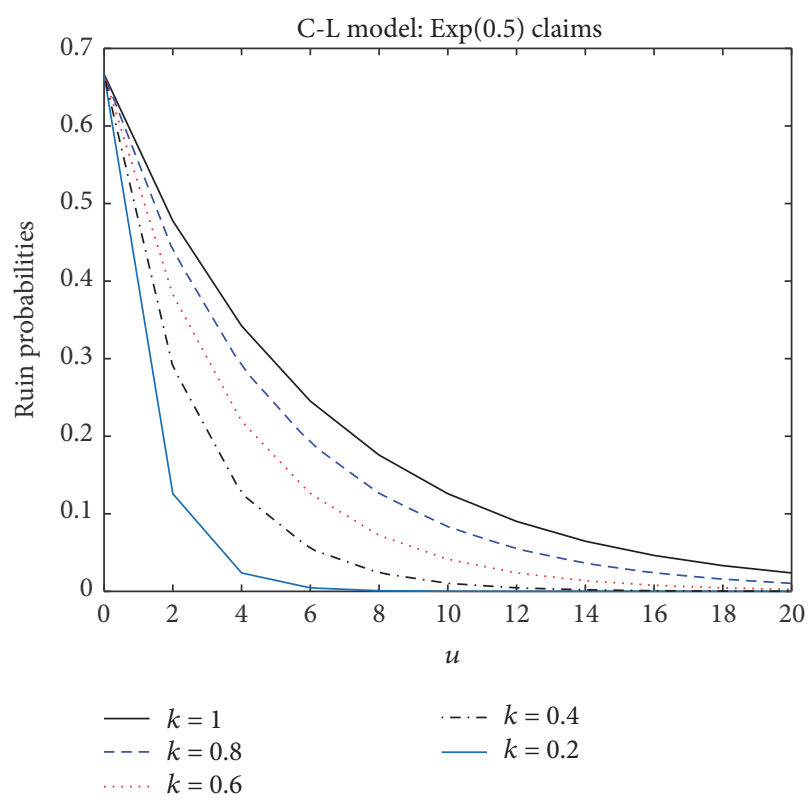

(a) $\operatorname{Exp}(0.5)$ claims

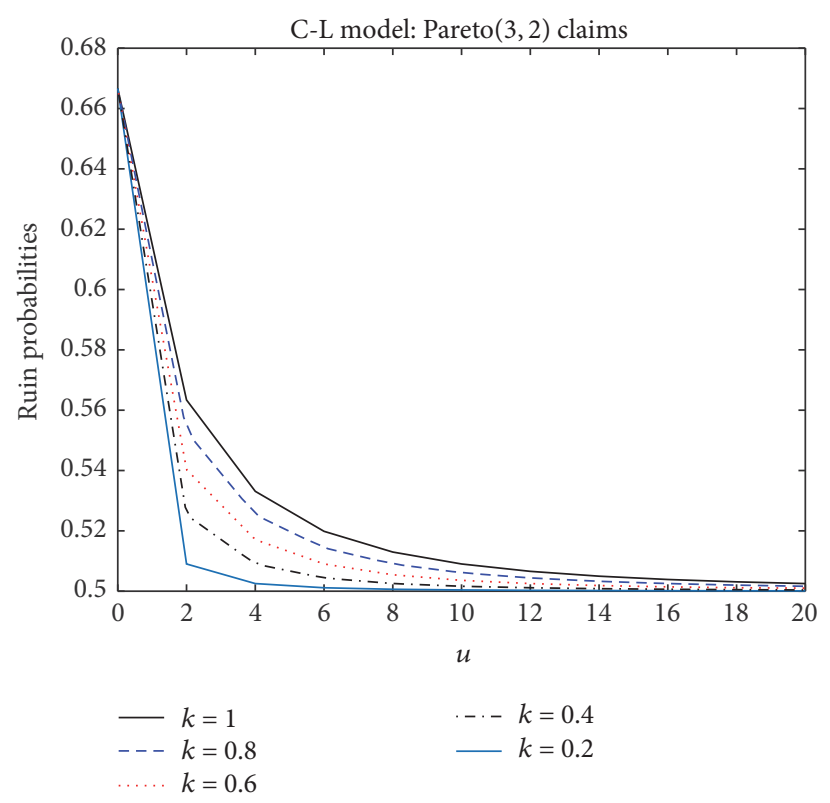

(b) Pareto $(3,2)$ claims

FIGURE 1: Ultimate ruin probabilities at different proportional retention levels in the Cramér-Lundberg model: $\lambda=2, c=6$.

which is a linear VIE of the second kind with $K(u, x)$ and $h(u)$ as given in (26).

\section{Numerical Results}

We solved (21) using the fourth-order block-by-block method, a full description of which can be found in $[11,14,15]$. $\operatorname{Exp}(\beta)$ refers to the exponential density $f(x)=\beta e^{-\beta x}$, so that the distribution function for the exponential distribution is $F(x)=1-e^{-\beta x}$ and its tail distribution is $\bar{F}(x)=1-$ $F(x)=e^{-\beta x}$. The mean excess function for the exponential distribution is $e_{F}(x)=1 / \beta$ and $G(x)=x-(1 / \beta) F(x)$. The $\operatorname{Pareto}(\alpha, \kappa)$ distribution, which is a special case of the threeparameter $\operatorname{Burr}(\alpha, \kappa, \tau)$ distribution, has density $f(x)=$ $\alpha \kappa^{\alpha} /(\kappa+x)^{\alpha+1}$ for $\alpha>0$ and $\kappa=\alpha-1>0$, and its distribution function is $F(x)=1-(\kappa /(\kappa+x))^{\alpha}$. The tail distribution of the Pareto distribution is $\bar{F}(x)=(\kappa /(\kappa+x))^{\alpha}$ and its mean excess function is $e_{F}(x)=1+x / \kappa$, so that $G(x)=x-(1+x / \kappa) F(x)$. A grid size of $h=0.01$ was used throughout. The data simulations were performed using a Samsung Series 3 PC with an Intel Celeron 847 processor at $1.10 \mathrm{GHz}$ and $6.0 \mathrm{~GB}$ RAM. To reduce computing time, the numerical method was implemented using the FORTRAN programming language, taking advantage of its DOUBLE PRECISION feature which gives a high degree of accuracy. The figures were constructed using MATLAB R2016a.

4.1. Ultimate Ruin Probability in the Cramér-Lundberg Model Compounded by Proportional Reinsurance. Here, the surplus process takes the form

$$
U_{t}^{\bar{R}}=u+k c t-\sum_{i=1}^{N_{t}} k X_{i}
$$

So, the survival probability $\phi(u)$ satisfies (21) and (22) with $a=\infty$ and $c^{\bar{R}}=k c$; that is, it satisfies a VIE of the second kind with kernel and forcing function given by

$$
\begin{aligned}
K(u, x) & =-\frac{\lambda \bar{F}(u-k x)}{k c} \\
h(u) & =\phi(0) .
\end{aligned}
$$

Figure 1 shows the ultimate ruin probabilities in the Cramér-Lundberg model for different proportional reinsurance retention levels $k$ and provides validity for the assertion that reinsurance does in fact reduce the ruin probability, thus increasing the insurance company's chances of survival. The results for the case $k=1$ (no reinsurance) are the same as those obtained in [14].

4.2. Ultimate Ruin Probability in the Cramér-Lundberg Model Compounded by Excess-of-Loss Reinsurance. This is the case of $k=1$ and $\sigma=0$, so the surplus process is

$$
U_{t}^{\bar{R}}=u+c^{\bar{R}} t-\sum_{i=1}^{N_{t}} X_{i} \wedge a,
$$

where $c^{\bar{R}}=c-(1+\theta) \lambda \mathbb{E}\left[\left(X_{i}-a\right)^{+}\right]$. Here, for the case $\underline{a}<$ $a<u$, the kernel and forcing function are given by

$$
\begin{gathered}
K(u, x)=-\frac{\lambda H(x, u)}{c^{\bar{R}}} \\
h(u)=\phi(0)
\end{gathered}
$$


TABLE 1: Ruin probabilities for XL reins. in CLM: $\operatorname{Exp}(0.5)$ claims $(\lambda=2, c=6)$.

\begin{tabular}{|c|c|c|c|c|c|}
\hline$u$ & $\psi_{\infty}(u)$ & $\psi_{35}(u)$ & $\psi_{30}(u)$ & $\psi_{25}(u)$ & $\psi_{20}(u)$ \\
\hline 0 & 0.6667 & 0.6667 & 0.6667 & 0.6667 & 0.6667 \\
\hline 2 & 0.4777 & 0.4777 & 0.4777 & 0.4777 & 0.4777 \\
\hline 4 & 0.3423 & 0.3423 & 0.3423 & 0.3423 & 0.3422 \\
\hline 6 & 0.2453 & 0.2453 & 0.2453 & 0.2453 & 0.2453 \\
\hline 8 & 0.1757 & 0.1757 & 0.1757 & 0.1757 & 0.1757 \\
\hline 10 & 0.1259 & 0.1259 & 0.1259 & 0.1259 & 0.1258 \\
\hline 12 & 0.0902 & 0.0902 & 0.0902 & 0.0902 & 0.0901 \\
\hline 14 & 0.0646 & 0.0646 & 0.0646 & 0.0646 & 0.0646 \\
\hline 16 & 0.0463 & 0.0463 & 0.0463 & 0.0463 & 0.0462 \\
\hline 18 & 0.0332 & 0.0332 & 0.0332 & 0.0332 & 0.0331 \\
\hline 20 & 0.0238 & 0.0238 & 0.0238 & 0.0238 & 0.0237 \\
\hline
\end{tabular}

TABLE 2: Ruin probabilities for XL reins. in CLM: $\operatorname{Par}(3,2)$ claims $(\lambda=2, c=6)$.

\begin{tabular}{llllll}
\hline$u$ & $\psi_{\infty}(u)$ & $\psi_{35}(u)$ & $\psi_{30}(u)$ & $\psi_{25}(u)$ & 0.6667 \\
\hline 0 & 0.6667 & 0.6667 & 0.6667 & 0.5639 \\
2 & 0.5634 & 0.5636 & 0.5637 & 0.5338 \\
4 & 0.5331 & 0.5335 & 0.5336 & 0.5206 & 0.5667 \\
6 & 0.5198 & 0.5202 & 0.5204 & 0.5138 & 0.5341 \\
8 & 0.5130 & 0.5134 & 0.5135 & 0.5099 & 0.5210 \\
10 & 0.5090 & 0.5095 & 0.5096 & 0.5074 & 0.5103 \\
12 & 0.5066 & 0.5070 & 0.5072 & 0.5058 & 0.5079 \\
14 & 0.5050 & 0.5054 & 0.5056 & 0.5048 & 0.5063 \\
16 & 0.5039 & 0.5043 & 0.5045 & 0.5040 & 0.5052 \\
18 & 0.5031 & 0.5036 & 0.5037 & 0.5034 \\
20 & 0.5025 & 0.5030 & 0.5032 & 0.5039 \\
\hline
\end{tabular}

with

$$
H(x, u)= \begin{cases}\bar{F}(u-x) & x<a \\ 1-(F(x+a)-F(a)) & x \geq a .\end{cases}
$$

This is simply (22) and (24) with $k=1$ and $c^{\bar{R}}=c-(1+$ $\theta) \lambda \mathbb{E}\left[\left(X_{i}-a\right)^{+}\right]$.

Ruin probabilities for the Cramér-Lundberg model compounded by excess-of-loss (XL) reinsurance are given in Table 1 for different values of the XL retention level $a$ ranging from 20 to infinity. Clearly, for $\operatorname{Exp}(0.5)$ claims, the ruin probabilities for the different retention levels reduce only very slightly as the retention level reduces. For Pareto(3,2) claims, the ruin probabilities increase slightly as the retention level reduces (as shown in Table 2), meaning that it is optimal not to reinsure. But comparing these probabilities with Figure 1 leads to the conclusion that proportional reinsurance results in much lower ruin probabilities for the CLM as well as the perturbed model.

4.3. Ultimate Ruin Probability in the Perturbed Classical Risk Process Compounded by Proportional Reinsurance. The survival probability $\phi(u)$ satisfies (21) and (26) with $a=\infty$; that is,

$$
\begin{aligned}
& \phi(u) \\
& +\frac{2}{\sigma^{2}} \int_{0}^{u}[k c-\lambda(u-k x)+\lambda G(u-k x)] \phi(x) d x \\
& =\frac{\sigma^{2}\left(\phi(0)+u \phi^{\prime}(0)\right)-2 k c u \phi(0)}{\sigma^{2}}
\end{aligned}
$$

which is a VIE of the second kind with kernel and forcing function given, respectively, by

$$
\begin{aligned}
K(u, x) & =\frac{2[k c-\lambda(u-k x)+\lambda G(u-k x)]}{\sigma^{2}} \\
h(u) & =u \phi^{\prime}(0) \quad \text { if } \sigma^{2}>0 .
\end{aligned}
$$

Figure 2 depicts the ruin probabilities for the diffusionperturbed model compounded by proportional reinsurance for different retention levels ranging from $k=1$ (no reinsurance) to $k=0.2$ ( $80 \%$ reinsurance). In the case of both $\operatorname{Exp}(0.5)$ claims and $\operatorname{Pareto}(3,2)$ claims, applying proportional reinsurance significantly reduces the ultimate ruin probability of an insurance company. 


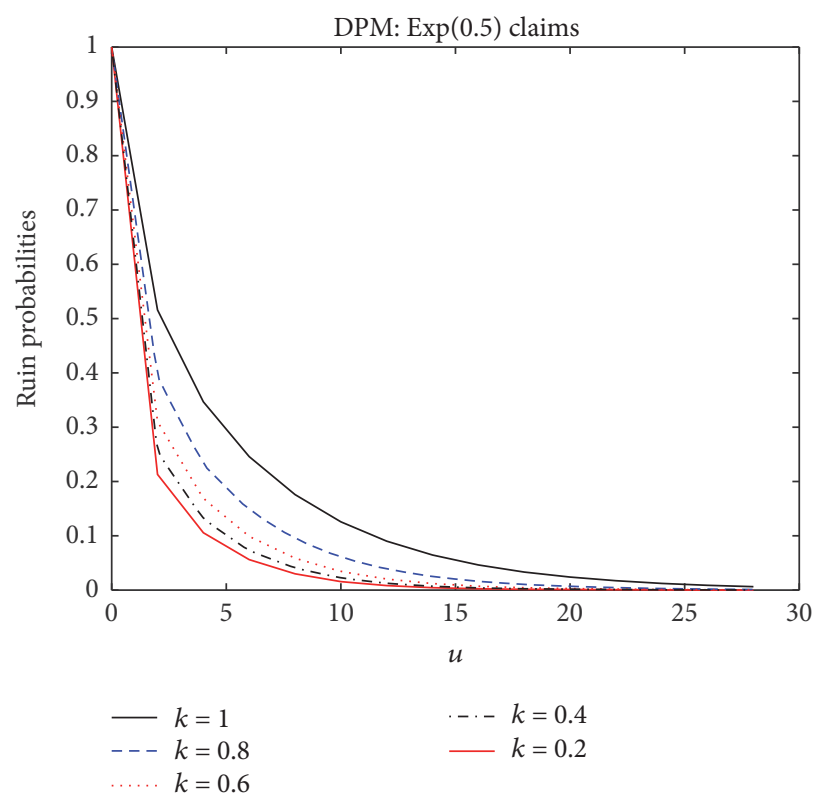

(a) $\operatorname{Exp}(0.5)$ claims

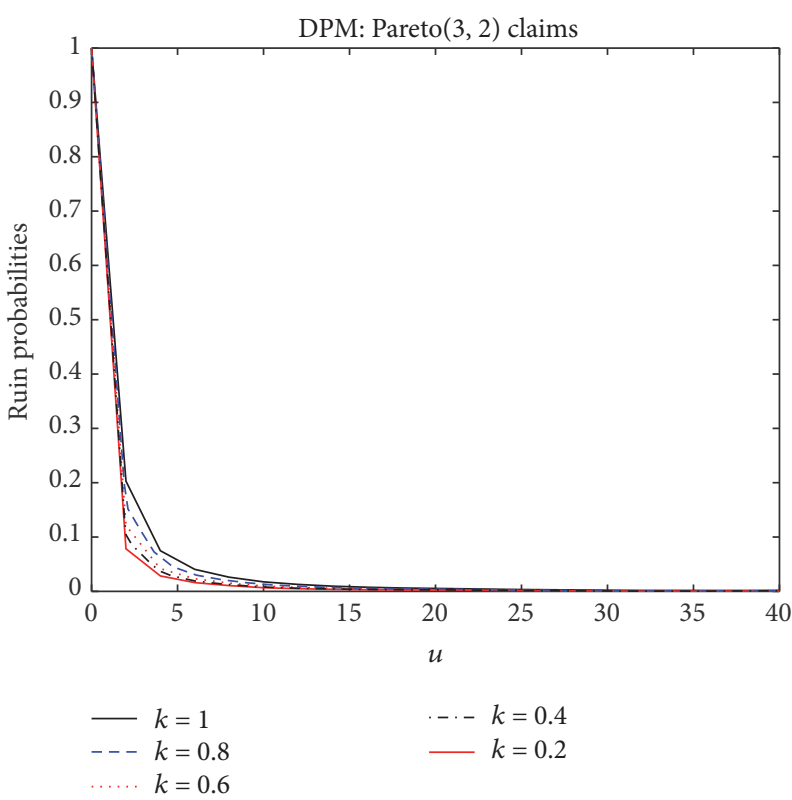

(b) Pareto (3, 2) claims

FIGURE 2: Ultimate ruin probabilities at different proportional retention levels in the diffusion-perturbed model: $\lambda=2, c=6, \sigma=0.02$.

TABLE 3: Ruin probabilities for XL reins. in DPM: $\operatorname{Exp}(0.5)$ claims $(\lambda=2, c=6, \sigma=0.02)$.

\begin{tabular}{llllll}
\hline$u$ & $\psi_{\infty}(u)$ & $\psi_{35}(u)$ & $\psi_{30}(u)$ & $\psi_{25}(u)$ & 1.0000 \\
\hline 0 & 1.0000 & 1.0000 & 1.0000 & 0.5155 & 0.3461 \\
2 & 0.5159 & 0.5159 & 0.5159 & 0.2451 \\
4 & 0.3467 & 0.3467 & 0.3466 & 0.1752 & 0.3109 \\
6 & 0.2458 & 0.2458 & 0.2457 & 0.1250 & 0.2380 \\
8 & 0.1759 & 0.1759 & 0.1758 & 0.0893 & 0.1674 \\
10 & 0.1257 & 0.1258 & 0.1257 & 0.0638 \\
12 & 0.0901 & 0.0901 & 0.0901 & 0.0455 \\
14 & 0.0646 & 0.0646 & 0.0645 & 0.0807 \\
18 & 0.0463 & 0.0463 & 0.0463 & 0.0550 \\
20 & 0.0333 & 0.0333 & 0.0332 & 0.0365 \\
\hline
\end{tabular}

4.4. Ultimate Ruin Probability in the Perturbed Classical Risk Process Compounded by Excess-of-Loss Reinsurance. The survival probability $\phi(u)$ satisfies a VIE of the second kind with kernel $K(u, x)$ as given in (23) (for the case $u \leq \underline{a}<a$ ) and (26) (for the case $\underline{a}<a<u$ ), with $k=1$, and forcing function $h(u)=u \phi^{\prime}(0)$ in both cases. That is,

$$
\begin{aligned}
& \text { for } u \leq \underline{a}<a, K(u, x)=2\left[c^{\bar{R}}+\lambda G(u-x)-\lambda(u-x)\right] / \sigma^{2} \\
& \text { for } \underline{a}<a<u, K(u, x)=2\left[c^{\bar{R}}+\lambda H_{2}(x, u)-\lambda(u-x)\right] / \sigma^{2}
\end{aligned}
$$

with

$$
H_{2}(x, u)= \begin{cases}G(u-x) & x<a \\ (F(x+a)-F(a))(u-x) & x \geq a .\end{cases}
$$

The impact of XL reinsurance on the ruin probabilities in a diffusion-perturbed model is evident from Table 3 which shows a reduction in the ruin probabilities for XL retentions not exceeding $a=30$ for small claims. However, as can be seen from Table 4, the ruin probabilities for large claims are higher for values of $a$ exceeding 150 but reduce significantly for values of $a$ below 150. But again, if we compare these results with Figure 2 we see that the ruin probabilities are much lower for proportional reinsurance.

4.5. Optimal Reinsurance Strategy: Asymptotic Ruin Probabilities. It is known that the optimal quota-share retention $k^{*}$ tends to the asymptotically optimal $k^{\rho}$ that maximizes the adjustment coefficient $\rho$ [13]. Therefore, since it was not possible to determine the optimal retention $k^{*}$ from the results discussed in Sections 4.1-4.4, we will use asymptotic ruin probabilities. For illustrative purposes, we will now find 
TABLE 4: Ruin probabilities for XL reins. in DPM: $\operatorname{Par}(3,2)$ claims $(\lambda=2, c=6, \sigma=0.02)$.

\begin{tabular}{llllll}
\hline$u$ & $\psi_{\infty}(u)$ & $\psi_{200}(u)$ & $\psi_{150}(u)$ & $\psi_{100}(u)$ & 1.0000 \\
\hline 0 & 1.0000 & 1.0000 & 1.0000 & 0.2022 & 0.0000 \\
2 & 0.2026 & 0.2029 & 0.2027 & 0.0740 & 0.1973 \\
4 & 0.0744 & 0.0747 & 0.0745 & 0.0397 & 0.0683 \\
6 & 0.0401 & 0.0405 & 0.0403 & 0.0252 & 0.0167 \\
8 & 0.0257 & 0.0260 & 0.0258 & 0.0119 & 0.0106 \\
10 & 0.0171 & 0.0174 & 0.0172 & 0.0088 & 0.0058 \\
12 & 0.0124 & 0.0127 & 0.0125 & 0.0067 & 0.0027 \\
14 & 0.0093 & 0.0096 & 0.0094 & 0.0054 \\
16 & 0.0072 & 0.0075 & 0.0073 & 0.0006 \\
20 & 0.0058 & 0.0061 & 0.0059 & 0.0042 \\
\hline
\end{tabular}

TABLE 5: Asympt. ruin prob. for CLM with proportional reins. (Pareto claims) $(c=6, \lambda=2, \theta=\eta=1$ ).

\begin{tabular}{|c|c|c|c|c|c|c|}
\hline$u$ & $\psi_{1}(u)$ & $\psi_{0.6}(u)$ & $\psi_{0.2}(u)$ & $\psi_{0.05}(u)$ & $\psi_{0.0125}(u)$ & $\psi_{0.003125}(u)$ \\
\hline 0 & 1.0000 & 1.0000 & 1.0000 & 1.0000 & 1.0000 & 1.0000 \\
\hline 2 & 0.3333 & 0.2308 & 0.0909 & 0.0244 & 0.0062 & 0.0016 \\
\hline 4 & 0.2000 & 0.1304 & 0.0476 & 0.0123 & 0.0031 & 0.0008 \\
\hline 6 & 0.1429 & 0.0909 & 0.0323 & 0.0083 & 0.0021 & 0.0005 \\
\hline 8 & 0.1111 & 0.0698 & 0.0244 & 0.0062 & 0.0016 & 0.0004 \\
\hline 10 & 0.0909 & 0.0566 & 0.0196 & 0.0050 & 0.0012 & 0.0003 \\
\hline 12 & 0.0769 & 0.0476 & 0.0164 & 0.0041 & 0.0010 & 0.0003 \\
\hline 14 & 0.0667 & 0.0411 & 0.0141 & 0.0036 & 0.0009 & 0.0002 \\
\hline 16 & 0.0588 & 0.0361 & 0.0123 & 0.0031 & 0.0008 & 0.0002 \\
\hline 18 & 0.0526 & 0.0323 & 0.0110 & 0.0028 & 0.0007 & 0.0002 \\
\hline 20 & 0.0476 & 0.0291 & 0.0099 & 0.0025 & 0.0006 & 0.0002 \\
\hline
\end{tabular}

the optimal strategies only in the CLM for both the small and large claim cases.

4.5.1. Exponential Claims. We note, as in [13], that for exponential claims the optimal choice of the quota-share retention $k$ that maximizes the adjustment coefficient $\rho(k)$ is given by

$$
k^{\rho}=\min \left\{\left(1-\frac{\eta}{\theta}\right)\left(1+\frac{1}{\sqrt{1+\theta}}\right), 1\right\},
$$

where $\theta$ and $\eta$ are, respectively, the safety loadings of the reinsurer and insurer. Because maximizing the adjustment coefficient yields the asymptotically best strategy, we expect that the optimal retention $k^{*}$ will tend to $k^{\rho}$. Since this study assumes cheap reinsurance (i.e., $\theta=\eta$ ), we have the fact that $k^{\rho}=0$. That is, it is optimal for the insurance company to reinsure the entire portfolio or to take full proportional reinsurance.

4.5.2. Pareto Claims. For a given initial surplus $u$ and a retention level $k \in[0,1]$, let the calculated ruin probability be given by $\psi_{k}(u)$. Then, for large claims, the asymptotic values of the ruin probability are given by

$$
\psi_{k}(u)=\frac{1}{k \theta-(\theta-\eta)} \frac{k}{1+u / k} .
$$

This ruin probability is minimized when $k^{\rho}=2(\theta-\eta) u /(\theta u-$ $(\theta-\eta))$. Thus, for Pareto-distributed claims, assuming $\theta=$ $\eta=1$, we find that $\psi_{k}(u)=k /(k+u)$ and that $k^{\rho}=0$ as well. The insurance company should reinsure the entire portfolio of risks. The results for different values of $k$ are summarized in Table 5 and shown in Figure 3.

It is clear from Figure 3 that the ruin probabilities become smaller as $k \rightarrow 0$, meaning that the asymptotically optimal retention must be $k^{\rho}=0$. This confirms the results shown in Figure 1. And since the optimal retention $k^{*}$ tends to the asymptotically optimal $k^{\rho}$ that maximizes the adjustment coefficient, it follows that $k^{*}=0$. This means that the insurance company must cede the entire portfolio of risks to a reinsurer. We can therefore conclude that the optimal combinational quota-share and $\mathrm{XL}$ reinsurance strategy is $\left(k^{*}, a^{*}\right)=(0, \infty)$. 


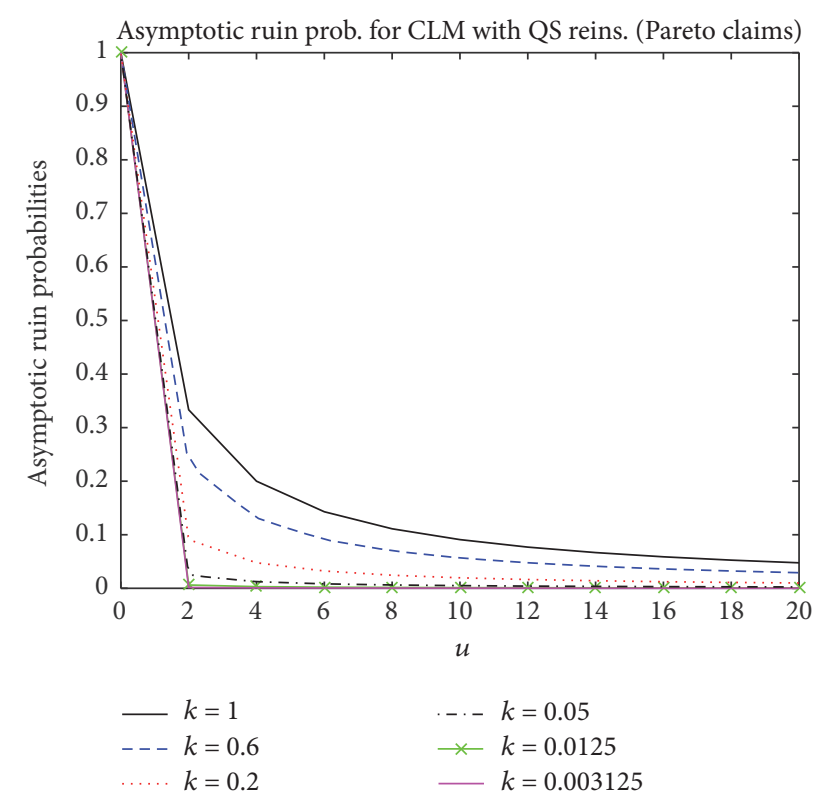

FIGURE 3: Asymptotic ruin probabilities for large claims in the CLM with proportional reinsurance $(c=6, \lambda=2, \theta=\eta=1)$.

\section{Conclusion}

While the results presented in the previous section show that proportional and XL reinsurance both result in a reduction in the ruin probabilities, the reduction is more drastic for Pareto than for exponential claims in both the Cramér-Lundberg and diffusion-perturbed models. On the one hand, a comparison of the figures presented in the foregoing shows that proportional reinsurance results in lower ruin probabilities than XL reinsurance and is therefore optimal. The optimal quota-share retention was found as $k^{*}=0$, meaning that in both the small and large claim cases in the CramérLundberg model, it is optimal for the insurance company to reinsure the whole portfolio using proportional reinsurance. Going by the results in Figure 3, the same conclusion can be drawn about the diffusion-perturbed model. Thus, the optimal combinational quota-share and XL reinsurance strategy is a pure quota-share reinsurance with $k^{*}=0$; that is, $\left(k^{*}, a^{*}\right)=(0, \infty)$. It should be noted that full reinsurance is not ideal from the reinsurer's standpoint and this provides a strong argument for the use of noncheap reinsurance.

On the other hand, the literature shows that the optimal reinsurance strategy is a pure XL, that is, $\left(1, a^{*}\right)$ (see, e.g., $[7,8,16])$. Possible extensions to the work are the inclusion of investments and dividend payouts as well as considering noncheap reinsurance, whereby, for a given risk, the reinsurer requires more premium and therefore uses a higher safety loading, than the insurer.

\section{Conflicts of Interest}

The authors declare that there are no conflicts of interest regarding the publication of this paper.

\section{Acknowledgments}

This work was supported by Mulungushi University, the Nelson Mandela African Institution of Science and Technology, and the Zambian Ministry of Higher Education through the Support to Science and Technology Education Project (SSTEP) funded by the African Development Bank Group. The authors wish to also thank Christian Kasumo whose M.S. thesis [15] provided some theoretical background on proportional reinsurance, ultimate ruin probabilities, and the numerical solution of Volterra integral equations.

\section{References}

[1] S. D. Promislow and V. R. Young, "Minimizing the probability of ruin when claims follow Brownian motion with drift," North American Actuarial Journal, vol. 9, no. 3, pp. 109-128, 2005.

[2] J. Kasozi, C. W. Mahera, and F. Mayambala, "Controlling ultimate ruin probability by quota-share reinsurance arrangements," International Journal of Applied Mathematics and Statistics, vol. 49, no. 19, pp. 1-15, 2013.

[3] C. S. Liu and H. Yang, "Optimal investment for an insurer to minimize its probability of ruin," North American Actuarial Journal, vol. 8, no. 2, pp. 11-31, 2004.

[4] C. Hipp and M. Plum, "Optimal investment for insurers," Insurance: Mathematics \& Economics, vol. 27, no. 2, pp. 215-228, 2000.

[5] H. Schmidli, "On minimizing the ruin probability by investment and reinsurance," The Annals of Applied Probability, vol. 12, no. 3, pp. 890-907, 2002.

[6] P. Li, M. Zhou, and C. Yin, "Optimal reinsurance with both proportional and fixed costs," Statistics \& Probability Letters, vol. 106, pp. 134-141, 2015.

[7] X. Hu and L. Zhang, "Ruin probability in a correlated aggregate claims model with common Poisson shocks: application to reinsurance," Methodology and Computing in Applied Probability, vol. 18, no. 3, pp. 675-689, 2016.

[8] X. Zhang and Z. Liang, "Optimal layer reinsurance on the maximization of the adjustment coefficient," Numerical Algebra, Control and Optimization, vol. 6, no. 1, pp. 21-34, 2016.

[9] B.-G. Jang and K. T. Kim, "Optimal reinsurance and asset allocation under regime switching," Journal of Banking \& Finance, vol. 56, pp. 37-47, 2015.

[10] R. Verlaak and J. Beirlant, "Optimal reinsurance programs. An optimal combination of several reinsurance protections on a heterogeneous insurance portfolio," Insurance: Mathematics \& Economics, vol. 33, no. 2, pp. 381-403, 2003.

[11] P. Linz, Analytical and Numerical Methods for Volterra Equations, vol. 7 of SIAM Studies in Applied Mathematics, Society for Industrial and Applied Mathematics (SIAM), Philadelphia, Pa, USA, 1985.

[12] L. Centeno, "On combining quota-share and excess of loss," ASTIN Bulletin, vol. 15, no. 1, pp. 49-63, 1985.

[13] H. Schmidli, Stochastic Control in Insurance, Probability and Its Applications (New York), Springer, London, UK, 2008.

[14] J. Paulsen, J. Kasozi, and A. Steigen, "A numerical method to find the probability of ultimate ruin in the classical risk model with stochastic return on investments," Insurance: Mathematics \& Economics, vol. 36, no. 3, pp. 399-420, 2005. 
[15] C. Kasumo, Minimizing the probability of ultimate ruin by proportional reinsurance and investment [M.S. thesis], University of Dar es salaam, 2011.

[16] D. Li, D. Li, and V. R. Young, "Optimality of excess-loss reinsurance under a mean-variance criterion," Insurance: Mathematics \& Economics, vol. 75, pp. 82-89, 2017. 


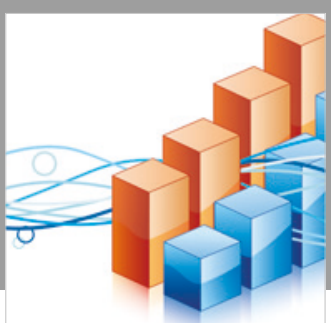

Advances in

Operations Research

\section{-n-m}
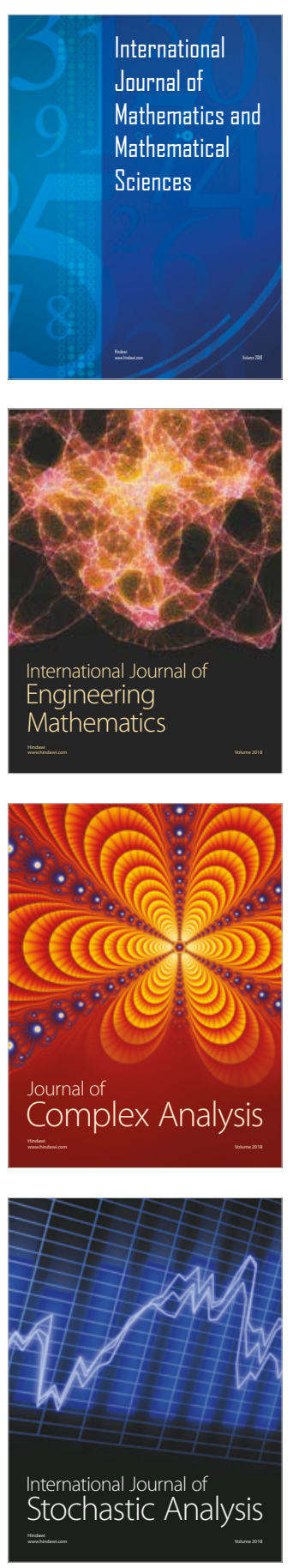
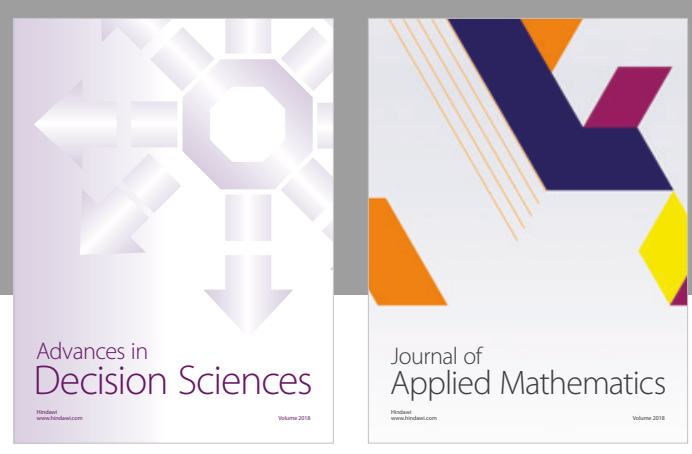

Journal of

Applied Mathematics
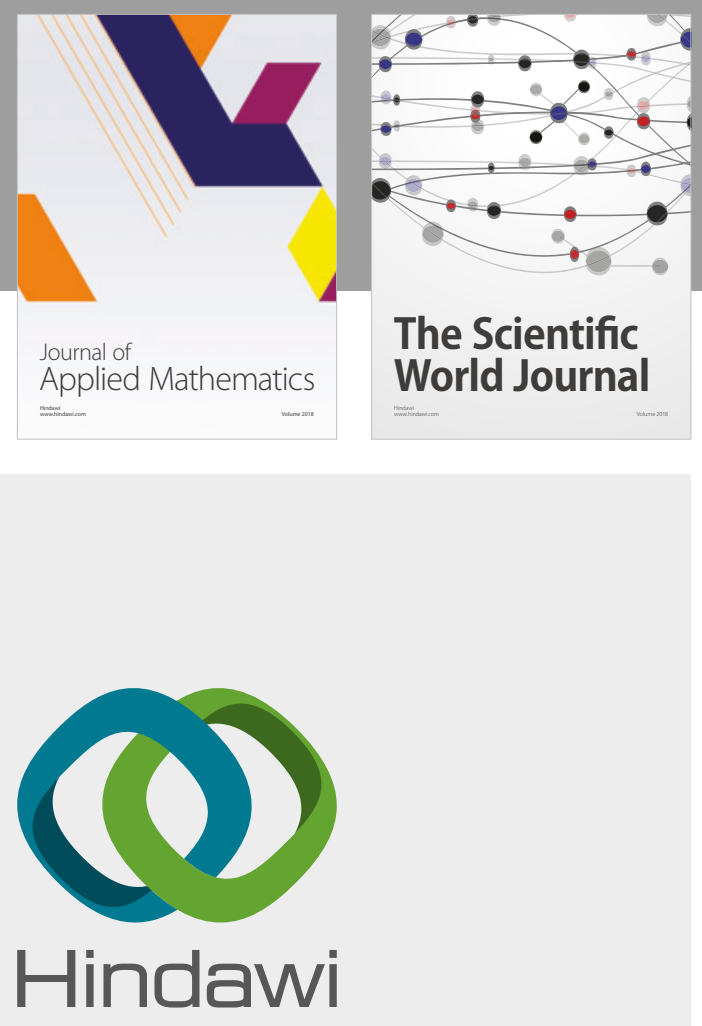

Submit your manuscripts at

www.hindawi.com

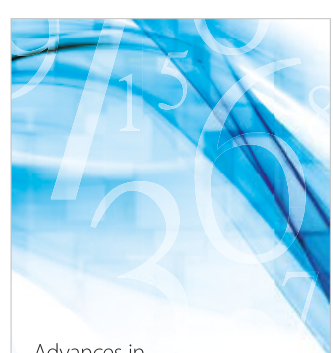

Advances in
Numerical Analysis
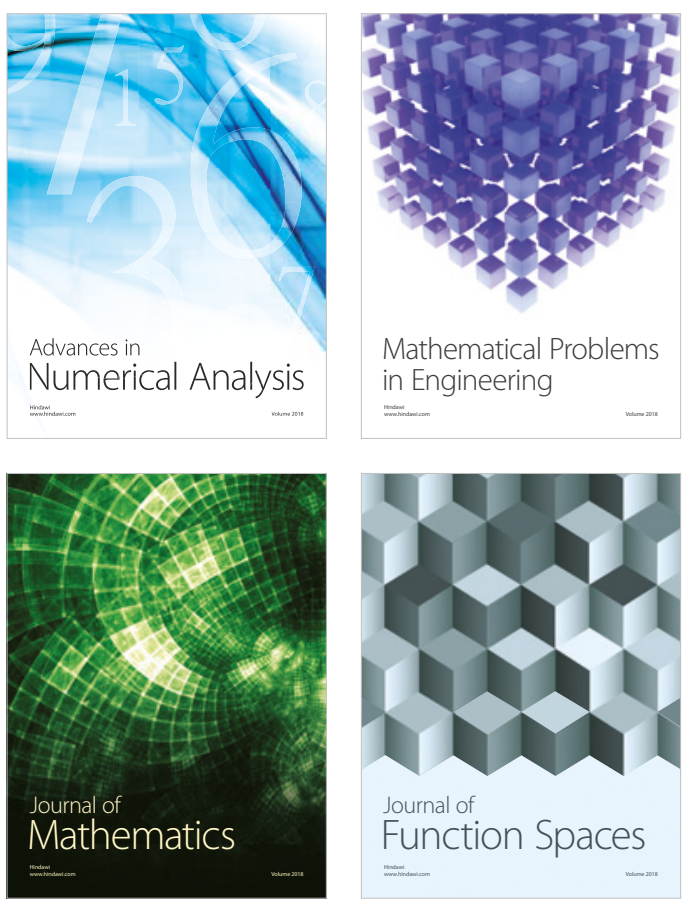

Mathematical Problems in Engineering

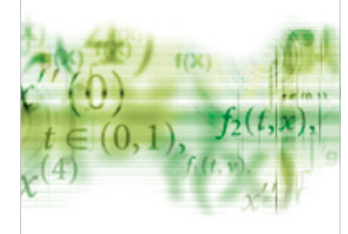

International Journal of

Differential Equations

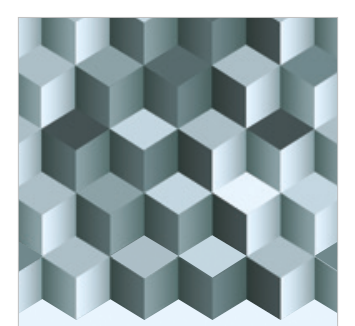

Journal of

Function Spaces

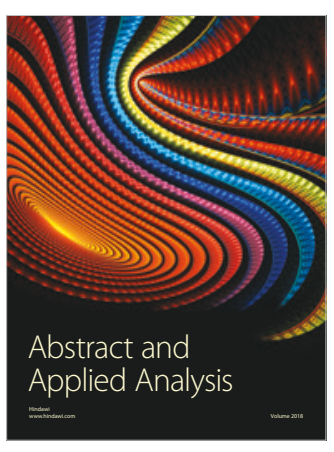

The Scientific

World Journal

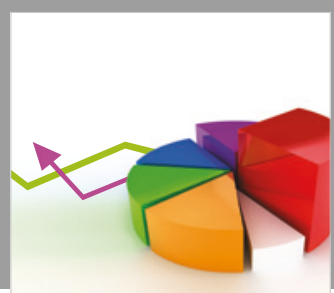

Journal of

Probability and Statistics
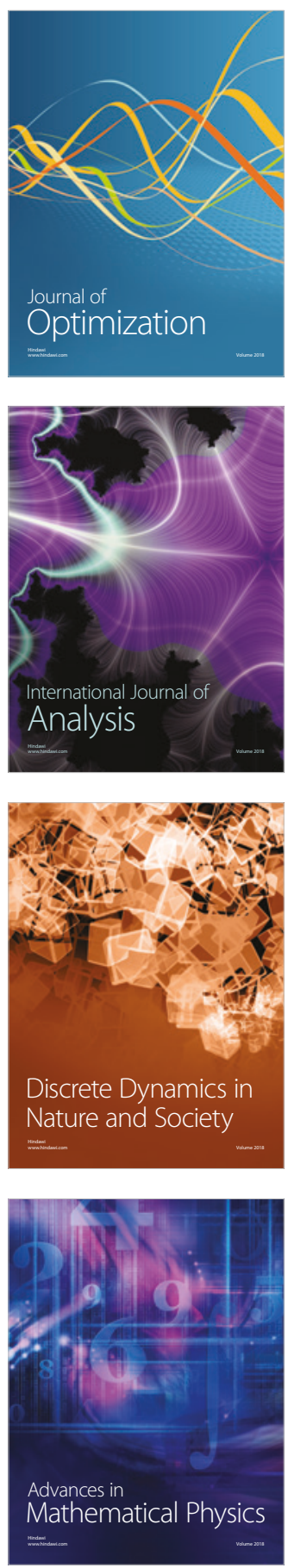